\title{
Prevalência e fatores de risco para tabagismo em adolescentes na América do Sul: uma revisão sistemática da literatura
}

\author{
Maura C. Malcon, ${ }^{1}$ Ana Maria B. Menezes, ${ }^{2}$ Maria de Fátima S. Maia, ${ }^{1}$ \\ Moema Chatkin ${ }^{2}$ e César G. Victora ${ }^{3}$
}

RESUMO Objetivo. Descrever a prevalência do tabagismo em adolescentes e os fatores associados ao tabagismo nesse grupo etário na América do Sul.

Métodos. Foram pesquisadas as bases de dados MEDLINE (1966-2002) e Literatura LatinoAmericana e do Caribe em Ciencias da Saúde ("Lilacs") (1982-2002), além de documentos governamentais e não-governamentais e sites na Internet. Foram identificados 315 artigos, dos quais 45 foram considerados relevantes para o presente trabalho.

Resultados. Em todos países estudados, foi grande a variação nos níveis de prevalência; tal variação dependeu, em parte, do grupo etário enfocado e da definição de fumante utilizada no estudo. O hábito de fumar entre irmãos e amigos foi o principal fator de risco para tabagismo na adolescência. O baixo rendimento escolar, a idade mais avançada, o sexo masculino, o trabalho remunerado e a separação dos pais também foram identificados como fatores de risco. Conclusão. São necessários mais estudos de base populacional representativos dessa faixa etária e critérios uniformes para definir os fumantes.

Palavras--chave Tabagismo, adolescência, América do Sul.

O tabaco é uma droga lícita largamente utilizada em todo o mundo. Atualmente, nos países desenvolvidos, é a principal causa de enfermidades evitáveis e de mortes prematuras (1-3). Considera-se que a nicotina, substância própria do cigarro, causa adição e tabaco-dependência $(2,4,5)$. Entre 33 e

1 Universidade Federal de Pelotas. Correspondência e pedidos de separatas devem ser enviados a Maura C. Malcon no seguinte endereço: Rua Félix da Cunha 916/1001, CEP 96010-000, Pelotas, RS, Brasil. E-mail: mmalcon@zaz.com.br

2 Universidade Federal de Pelotas, Departamento de Clínica Médica.

3 Universidade Federal de Pelotas, Departamento de Medicina Social.
$50 \%$ dos adolescentes nor-te-americanos que experimentaram o cigarro tornaramse drogaditos (6); aproximadamente $90 \%$ dos adultos fumantes adquiriram o vício na adolescência $(7,8)$. Nos últimos anos, a indústria do tabaco tem concentrado seus esforços de venda nos adolescentes, visto que estes serão os novos consumidores $(6,7,9)$.

A revisão sistemática da literatura aqui apresentada tem como objetivo delinear o panorama do tabagismo na adolescência em países da América do Sul, com ênfase na prevalência e fatores de risco para tabagismo em adolescentes.

\section{MATERIAIS E MÉTODOS}

A revisão sistemática da literatura foi realizada a partir das bases de dados on-line Medline (1966-2002) (10) e Lilacs (1982-2002) (11), utilizando os seguintes descritores: tabagismo, adolescência, criança, América do Sul. Os descritores em inglês foram: smoking, adolescence, child, South America. A revisão foi ampliada através de busca em outras fontes, tais como documentos governamentais (12-14) e não governamentais (15), estatísticas de saúde (14), sites na Internet sobre tabagismo $(13,16)$, referências citadas nos artigos obtidos e contatos com autores. O total de 
artigos obtidos através dessa busca foi de 315.

Os artigos selecionados preenchiam os seguintes critérios: população com idade de 10 a 19 anos, amostra representativa da população escolar ou da população em geral (também foram selecionados os estudos sobre tabagismo em adultos que incluíssem adolescentes); ter sido realizados em país da América do Sul e na zona urbana; conter definição clara de "fumante"; ter sido publicados em português, inglês ou espanhol; incluir resumo; ter como foco o uso de tabaco na forma de cigarros. $\mathrm{O}$ total de artigos selecionados, atendendo a esses critérios, foi de 45 .

\section{RESULTADOS}

\section{Prevalência de tabagismo em adolescentes}

No Brasil foram encontrados 16 estudos, sendo que 11 utilizaram delineamento transversal de base escolar e cinco de base populacional. De todos os demais países da América do Sul, obtiveram-se 29 estudos com delineamento transversal, sendo 19 de base escolar e 10 de base populacional. Desses 29 estudos, 13 foram do Chile.

Na tabela 1 estão listados os estudos sobre tabagismo entre escolares na América do Sul. No Brasil, as freqüências variaram de 3 a 15,8\% (12, 17-21), com ampla diversidade na definição de fumante e na faixa etária. Nos demais países da América do Sul, houve predominância de publicação de estudos no Chile. Os estudos que utilizaram o "uso diário" como definição para fumante (embora em diferentes faixas etárias) apontaram prevalências variando de 10,6 a 58,3\% (22-25).

A tabela 2 mostra os estudos de base populacional sobre tabagismo na América do Sul. No período de 1989 a 2000, no Brasil, foram realizados cinco estudos de base populacional. No Chile, quatro estudos populacionais foram realizados (13) utilizando o mesmo critério para fumante e faixa etária.

\section{Fatores de risco para tabagismo em adolescentes}

Os fatores de risco identificados foram: sexo, idade do adolescente, nível socioeconômico, rendimento escolar, presença de tabagismo nos pais, nos irmãos e amigos, separação dos pais e trabalho (tabela 3). O sexo masculino apresentou-se como fator de risco em seis estudos na América do Sul $(18,20,21,28,35,37)$, enquanto o sexo feminino foi apontado em três estudos $(23,25,27)$. Horta et al. (44) e Malcon (41), no Brasil, e Ivanovic et al. (22), no Chile, não demonstraram diferenças significativas entre os sexos. Não há, portanto, uma consistência entre os estudos quanto à diferença de prevalência entre os sexos.

A maioria dos estudos mostrou que o aumento da prevalência de tabagismo entre os adolescentes está diretamente associado à idade (20-23, 27, 30, 31, 41). Malcon (41), mediante análise multivariada, demonstrou razão de chances (odds ratio, OR) para tabagismo de 9,9 no grupo de 14 a 16 anos e de 28,7 no grupo de 17 a 19 anos, em relação aos jovens 10 a 13 anos.

Na literatura, o hábito de fumar dos pais foi, freqüentemente, identificado como fator de risco para tabagismo na adolescência, embora nem sempre isso tenha sido demonstrado. Barbosa et al. (20) observaram associação com o hábito de fumar em ambos os pais, e outros autores com hábito em um dos pais $(18,27)$. Muza e Costa (45), em Brasilia, não demonstraram associação entre tabagismo na adolescência e tabagismo dos pais. No estudo de Malcon (41), após controle dos fatores de confusão, o hábito de fumar da mãe revelou OR de 1,6 para tabagismo na adolescência, mas o valor de $P$ ficou no limiar da significância $(P$ $=0,06$ ). Tabagismo da mãe, no estudo de Segat et al. (29), em Santa Maria (Estado do Rio Grande do Sul), resultou em risco relativo (RR) de 1,8 para desenvolvimento de tabagismo na adolescência em comparação com adolescentes cujas mães não fumavam.

Tabagismo entre os amigos ou entre os irmãos foi apontado como fator de risco para hábito de fumar em adolescentes na maioria dos estudos $(22,27,29,35,36,41)$. Malcon (41) mostrou OR de 2,4 quando havia irmãos fumantes; de 4,0 para adolescentes com até dois amigos fumantes; e de 17,5 para aqueles com três ou mais amigos fumantes, tomando como grupo de referência os adolescentes sem amigos fumantes. O estudo de Segat et al. (29) apontou um RR de 2,3 para ter irmão fumante em relação a não ter irmão fumante, e RR de 5,2 para ter pelo menos um melhor amigo fumante em comparação com não ter nenhum amigo fumante. $\mathrm{O}$ tabagismo dos amigos esteve associado a um RR de 9,8 em relação a não ter amigos fumantes no estudo de Ivanovic et al. (22), em Santiago, Chile. No Equador, Padgett et al. (36), demonstraram um RR de 8,2 para menina com irmã fumante e RR de 1,6 para menino com irmão fumante, em comparação a não ter irmãos fumantes. No mesmo estudo, ter amigos fumantes mostrou RR de 5,4 para o sexo feminino e RR de 2,5 para o sexo masculino em comparação a não ter amigos fumantes.

A associação entre o tabagismo e o nível socioeconômico não demons-trou resultados consistentes. O nível socioeconômico alto foi fator de risco para tabagismo no estudo de Ivanovic et al. (22), no Chile, e no de Londoño (35), na Colômbia; no Brasil, o nível socioeconômico baixo foi fator de risco para hábito de fumar no estudo de Muza e Costa (45), em Brasilia, enquanto que, em Ribeirão Preto, Muza et al. (46) não observaram associação entre o nível socioeconômico e o hábito de fumar. Malcon (41) e Horta et al. (44), em Pelotas, também não demonstraram associação entre o tabagismo e o nível socioeconômico, após o controle para fatores de confusão.

A repetência escolar foi apontada por Schio et al. (27), em Porto Alegre, Brasil, como fator de risco para tabagismo na adolescência. Na Colômbia (35), após análise multivariada, a OR para a associação entre o hábito de fumar e o baixo rendimento escolar foi de 1,7, tendo como referência ter bom rendimento escolar.

Em Pelotas, Malcon (41) registrou OR de 3,5 para tabagismo em adolescentes com 0 a 4 anos de escolaridade comparados a adolescentes com 9 anos ou mais de escolaridade, através de regressão logística. Esse estudo demonstrou uma prevalência de tabagismo de 36,2\% entre adolescentes que não freqüentavam a escola. Nos que freqüentavam a escola, a prevalência de tabagismo foi de 7,7\%. Horta et al. (44), na 
TABELA 1. Estudos de base escolar sobre prevalência de tabagismo em adolescentes na América do Sul, 1966 a 2002

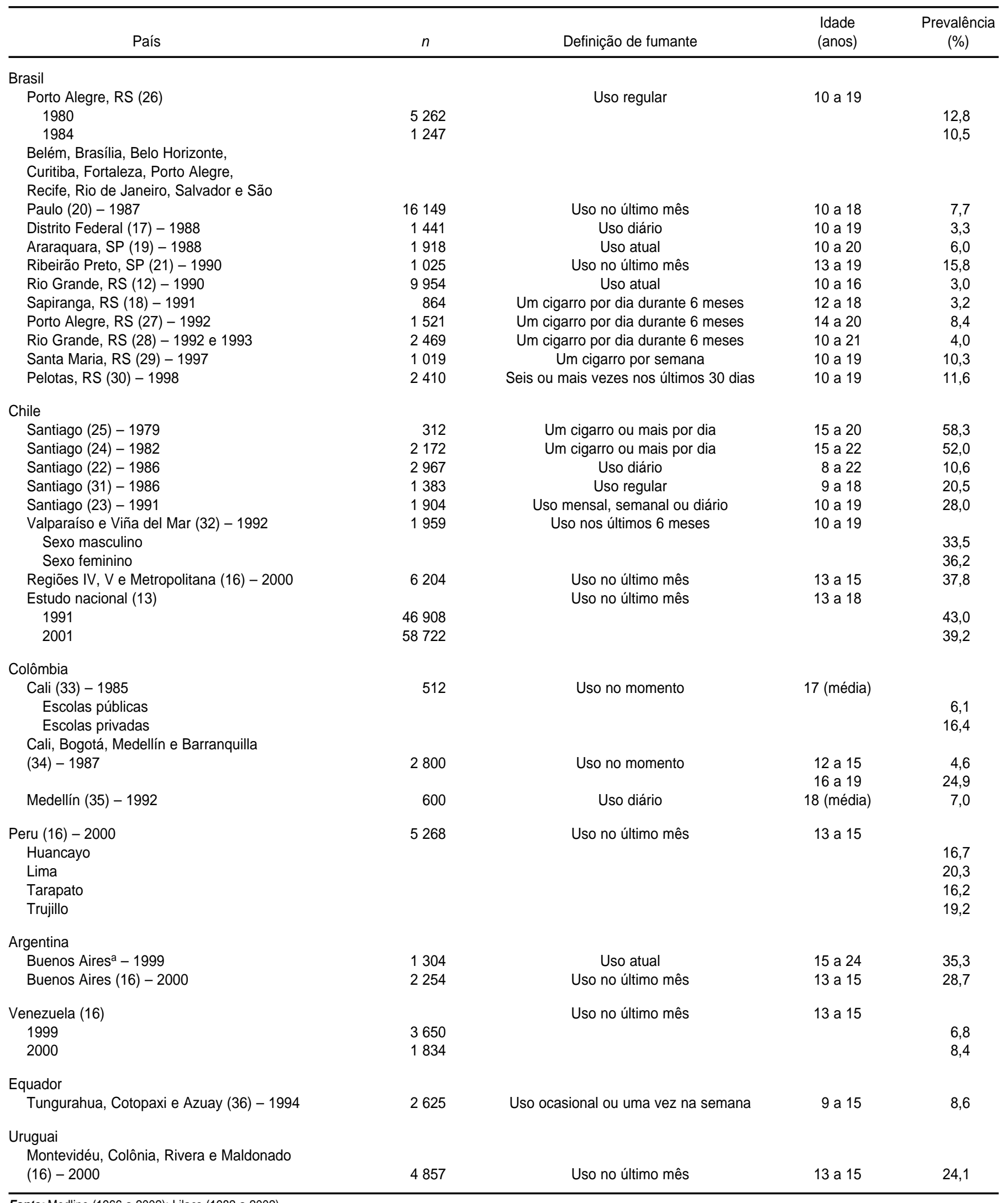

Fonte: Medline (1966 a 2002); Lilacs (1982 a 2002).

a Conforme comunicação pessoal de Isidoro Hasper, Secretaria de Salud, Prefeitura de Buenos Aires. 
TABELA 2. Estudos de base populacional sobre prevalência de tabagismo em adolescentes na América do Sul, 1966 a 2002

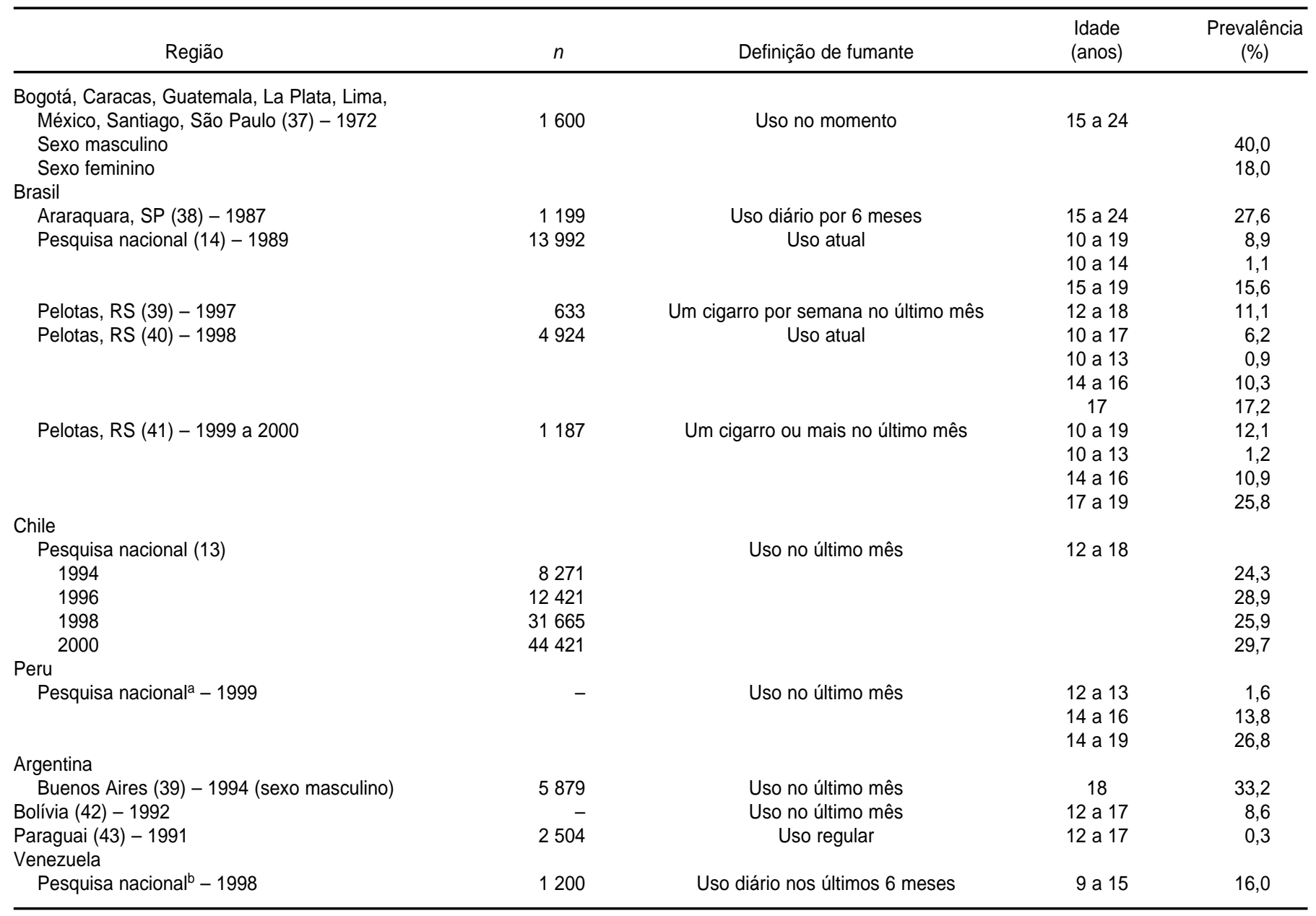

Fonte: Medline (1966 a 2002); Lilacs (1982 a 2002).

a Conforme comunicação pessoal de Alfonso Zavaleta Martínez-Vargas, Centro de Prevención y Educación para la Prevención del Abuso de Drogas (CEDRO), Lima, Peru.

b Conforme comunicação pessoal de Natasha Herrera, Organización Panamericana de la Salud.

mesma cidade, apontaram OR de 7,4 para adolescentes que não estavam estudando, tendo como referência os jovens que freqüentavam a escola. Em três estudos (20, $27,45)$, o trabalho remunerado foi apontado como fator de risco para tabagismo em jovens.

Além disso, Muza e Costa demonstraram associação entre o hábito de fu-mar e a separação dos pais (45), enquanto que a prática de esportes e a religião foram apontados como fatores de proteção para tabagismo na adolescência (45). Malcon (41) não encontrou associação entre a prática de esporte e o tabagismo em adolescentes, após análise multivariada.

\section{DISCUSSĀO}

A diversidade entre as definições utilizadas para a medida do desfecho (tabagismo em adolescentes) e as diferentes faixas etárias empregadas nos diversos estudos analisados foram limitações metodológicas importantes que impediram estabelecer uma evolução temporal para a freqüência de fumo em adolescentes no Brasil e demais países da América do Sul, assim como limitaram a comparação entre os estudos. Houve dificuldade em obter pesquisas de âmbito nacional em países fora do Brasil, por serem poucas ou por não estarem disponíveis nas bases de dados eletrônicas.

Os estudos em escolares no Brasil que utilizaram como definição para fumante o "uso regular" de cigarros e o "uso no último mês" mostraram prevalências consistentes entre 10\% e 16\%; já com a definição "uso de um cigarro por dia nos últimos 6 meses", as prevalências relatadas foram inferiores. No Chile, com o critério "uso regular" ou "uso no último mês" o percentual de escolares fumantes variou de 20 a $43 \%$, prevalências superiores às relatadas para o Brasil. No Brasil, as pesquisas de base populacional que utilizaram o critério "uso nos 
TABELA 3. Fatores de risco associados ao tabagismo em adolescentes, América Latina, 1966 a 2002

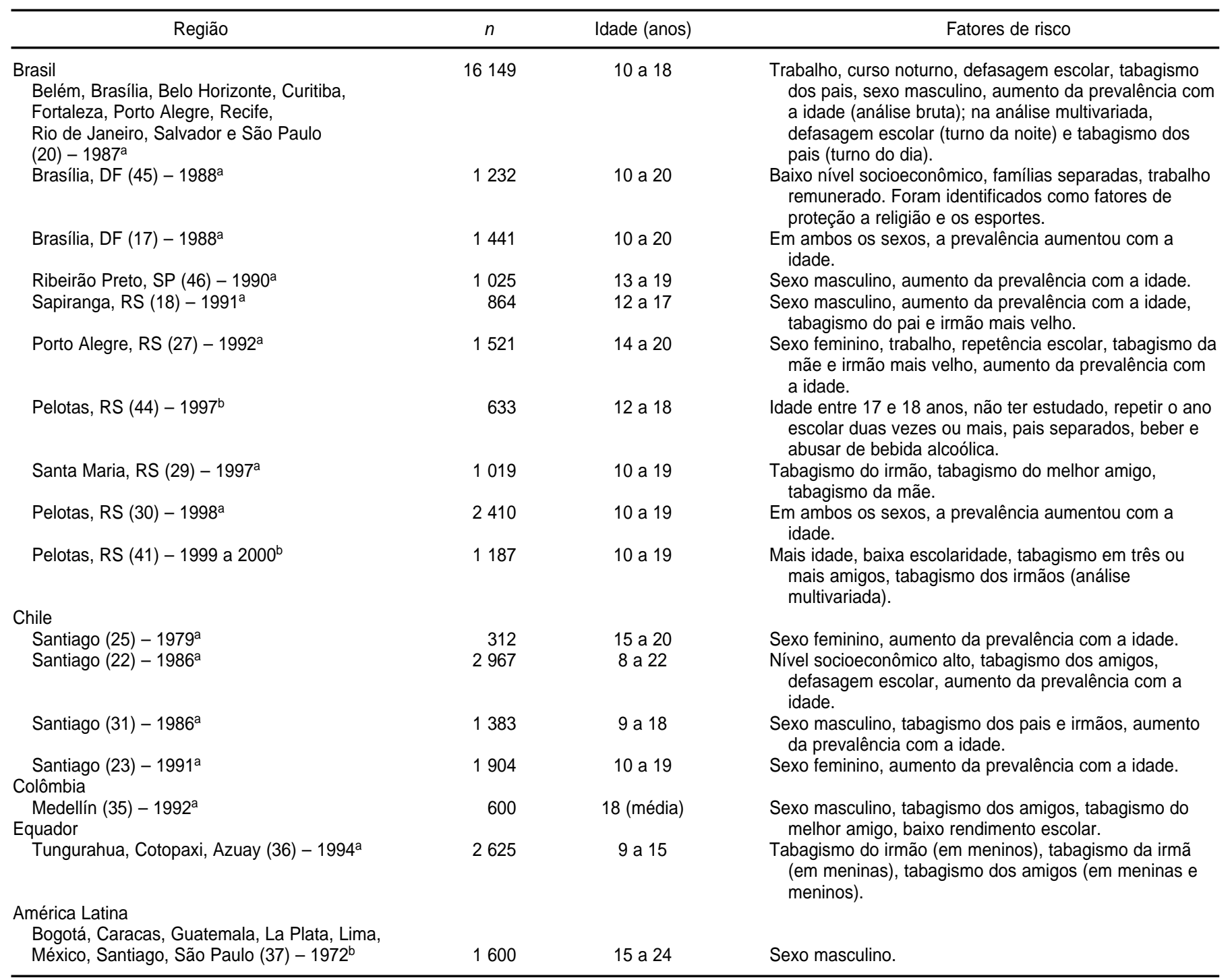

Fonte: Medline (1966 a 2002); Lilacs (1982 a 2002).

a Estudos de base escolar.

${ }^{\mathrm{b}}$ Estudos de base populacional.

últimos 30 dias" para definir o fumante mostram uma prevalência de 11,1 a $12,1 \%$ para tabagismo entre adolescentes, enquanto que, no Chile, a prevalência observada por esse tipo de estudo foi de $29,7 \%$.

Dos 17 estudos na América do Sul que avaliaram fatores de risco para tabagismo em jovens, somente cinco realizaram análise multivariada para controle de fatores de confusão $(20,22,35,41,44)$. Cabe ainda lembrar que o viés de publicação sempre pode afetar os resultados dos artigos de revisão; ou seja, estudos com achados negativos como a falta de associação entre o tabagismo na adolescência e alguns de seus fatores determinantes podem não ter sido publicados na literatura, de forma que tais resultados negativos não estariam sendo considerados na avaliação do todo.

A maioria dos estudos sobre tabagismo na adolescência utilizou questionários como instrumento para medir o desfecho, tanto em escolas como em residências. Embora muitos estudos tenham aplicado os questionários de forma confidencial e individual, não se pode descartar a possibilidade de o adolescente ter ocultado o fato de fumar. A utilização de marcadores biológicos pode ser usada para validar questionários $(47,48)$, mas, devido ao alto custo dessas medidas, a maioria dos estudos não as utiliza. 
De qualquer forma, se houve omissão de respostas afirmativas por parte desses adolescentes, a prevalência real pode ainda ser maior do que a encontrada.

\section{CONCLUSÕES}

A realização de pesquisas multicêntricas ou regionais de base populacional utilizando critérios uniformes para definir fumantes e faixas etárias padronizadas para os adolescentes permitirá acompanhar a evolução temporal do tabagismo neste grupo e a comparação entre os estudos. Sugere-se a utilização de critérios múltiplos (por exemplo, hábito de fumar diário nos últimos 6 meses, hábito de fumar alguma vez na vida, hábito de fumar no último mês) para permitir a comparabilidade com estudos passados.

A revisão bibliográfica mostrou que as pesquisas devem melhorar a qualidade metodológica, incluindo análise com ajuste para os fatores de confusão para identificar fatores de risco. A dosagem de marcadores biológicos como a cotinina seria útil na validação dos questionários aplicados em adolescentes.

Campanhas preventivas enfocando os malefícios do tabagismo na adolescência são necessárias, assim como a monitorização periódica do efeito de tais campanhas através de pesquisas de base populacional. As entidades médicas e os órgãos de saúde devem garantir a aplicação das leis antitabaco.

\section{REFERÊNCIAS}

1. Roemer R. Acción legislativa contra la epidemia mundial de tabaquismo. $2^{\mathrm{a}}$ ed. Ginebra: Organización Mundial de la Salud; 1995.

2. Jha P, Chaloupka FJ. Curbing the epidemic: governments and the economics of tobacco control. Washington, D.C.: World Bank; 1999.

3. Department of Health and Human Services. Reducing tobacco use: a report of the Surgeon General. Atlanta, Georgia: Centers for Disease Control and Prevention; 2000. Disponível em http://www.cdc.gov/tobacco/sgr_tobacco_ use.htm. Acessado em dezembro de 2002.

4. Silva VLC. Tabagismo: um problema de saúde pública no Brasil. J Bras Medicina 1990;59(2): 14-24.

5. Rosemberg J. Tabagismo e doenças pulmonares. Em: Tarantino AB, ed. Doenças pulmonares. $4^{a}$ ed. Rio de Janeiro: Guanabara Koogan; 1997. Pp. 189-200.

6. Elders MJ, Perry CL, Eriksen MP, Giovino GA. The report of the Surgeon General: preventing tobacco use among young people. Am J Public Health 1994;84(4):543-547.

7. World Health Organization (WHO). International consultation on tobacco and youth: what in the world works? Singapore: WHO; 1999

8. Giovino GA. Epidemiology of tobacco use among US adolescents. Nicotine Tob Res 1999; 1(Suppl 1):S31-S40.

9. Hijjar MA, Silva VLC. Epidemiologia do tabagismo no Brasil. J Bras Med 1991;60(1/2): 50-71.

10. National Library of Medicine. MEDLINE [site da Internet]. Disponível em http://www. ncbi.nlm.nih.gov/entrez/query.fcgi. Acessado em dezembro de 2002.

11. Biblioteca Regional de Medicina (BIREME). Base de dados LILACS [site da Internet]. Disponível em http://www.bireme.br. Acessado em dezembro de 2002.

12. Rio Grande do Sul, Secretaria de Justiça e da Segurança, Conselho Estadual de Entorpecentes (CONEN). Plano estadual de prevenção contra o uso do álcool, tabaco e outras drogas. Porto Alegre: CONEN; 1996.
13. Consejo Nacional para el Control de Estupefacientes (CONACE). Estudios nacionales sobre consumo de drogas en la población general de Chile. Disponível em http://www.conace. gov.cl. Acessado em dezembro de 2002.

14. Ministério da Saúde, Instituto Nacional de Alimentação e Nutrição (INAN). Pesquisa nacional sobre saúde e nutrição (PNSN): estatísticas sobre hábitos de fumo no Brasil. Brasília: INAN; 1989.

15. Associação Médica Brasileira, Comissão de Combate ao Tabagismo. Índice bibliográfico brasileiro sobre tabagismo. $2^{\mathrm{a}}$ ed. São Paulo: Associação Médica Brasileira; 1996.

16. World Health Organization (WHO), Centers for Disease Control and Prevention (CDC). Global youth tobacco survey (GYTS). Disponível em http://www.cdc.gov/tobacco/global/ GYTS.htm. Acessado em dezembro de 2002.b

17. Godoi AMM, Muza GM, Costa MP, Gama MLT. Consumo de substâncias psicoativas entre estudantes de rede privada. Rev Saude Publica 1991;25(2):150-156.

18. Bordin R, Nipper VB, Silva JO, Bortolomiol L. Prevalência de tabagismo entre escolares em município de área metropolitana da Região Sul, Brasil, 1991. Cad Saude Publica 1993;9(2): 185-189.

19. Simões MJS. Tabagismo entre estudantes do primeiro e segundo graus em Araraquara, $\mathrm{SP}$, Brasil, 1988. Medicina (Ribeirão Preto) 1990; 23(4):223-231.

20. Barbosa MTS, Carlini-Coltrin B, Silva Filho AR. $\mathrm{O}$ uso de tabaco por estudantes de primeiro e segundo graus em dez capitais brasileiras: possíveis contribuições da estatística multivariada para compreensão do fenômeno. Rev Saude Publica 1989;23(5):401-409.

21. Muza GM, Bettiol H, Muccillo G, Barbieri M. Consumo de substâncias psicoativas por adolescentes escolares de Ribeirão Preto, SP (Brasil): I. Prevalência do consumo por sexo, idade e tipo de substância. Rev Saude Publica 1997; 31(1):21-29.

22. Ivanovic DM, Castro CG, Ivanovic RM. Factores que inciden en el hábito de fumar de escolares de educación básica y media de Chile. Rev Saude Publica 1997;31(1):30-43.

23. Florenzano Urzua R. Risk factors and youth: the role of family and community. J Adolesc Health 1993;14(8):619-625.

24. Cabrera Reyes F, Salomón Rex C, López Bravo I, Vidal Oyarzún R. El hábito de fumar en estudiantes de educación media, en Santiago, Chile. Bol Oficina Sanit Panam 1982;93(6):533540.

25. Salas I, Ramos E, Peters G, Pesenti L, O'Ryan F, Nieme EB, et al. Prevalencia de tabaquismo en adolescentes del tercer año de la enseñanza media. Rev Med Chil 1982;110(12):1237-1244.

26. Achutti AC. Tabagismo entre escolares do Rio Grande do Sul. $2^{\mathrm{a}}$ ed. Porto Alegre: Organização Pan-Americana da Saúde; 1986.

27. Schio C, Reverbel E, Fernandes E, Gugel F, Kessler JB, Silva RC, et al. O tabagismo entre estudantes secundaristas da zona urbana de Porto Alegre. Rev HCPA 1992;12(2):117-120.

28. Halty L, Hüttner M, Neto I. Epidemiology of asthma, rhinitis and cigarette smoking in grade school students. XVII World Congress on Diseases of the Chest. Chest 1993;103(3): 270s.

29. Segat FM, Santos RP, Guillande S, Pasqualotto AC, Benvegnú LA. Fatores de risco associados ao tabagismo em adolescentes. Adolesc Latinoam 1998;1(3):163-169.

30. Tavares BF. Uso de drogas em adolescentes escolares em Pelotas, RS. Rev Saude Publica 2001;35(2):150-158.

31. Olivari F, de la Fuente M, López I. Smoking among elementary school children. Study in a population of low socioeconomic level. Rev Med Chil 1989;117(8):861-866.

32. Gutiérrez M, Rioseco F, Rojas A, Casanova D, Cordero M, Schiaffino M. Prevalencia de tabaquismo en la población general de Valparaíso y Viña del Mar. Rev Med Chil 1995;123(2): 250-256.

33. Bergonzoli Peláez G, Rico O, Ramírez A, Paz MI, Ramírez J, Rivas JC, et al. Uso de drogas entre estudiantes de Cali, Colombia. Bol Oficina Sanit Panam 1989;106(1):22-31. 
34. Torres de Galvis Y, Murrelle L. Consumo de substancias que producen dependencia en Colombia. Bull Pan Am Health Organ 1990;24(1): 12-21.

35. Londoño FJL. Factores relacionados con el consumo de cigarrillos en escolares adolescentes de la ciudad de Medellín. Bol Oficina Sanit Panam 1992;112(2):131-137.

36. Padgett D, Selwyn B, Kelder S. Ecuadorian adolescents and cigarette smoking: A crosssectional survey. Rev Panam Salud Publica 1998;4(2):87-92.

37. Joly DJ. Cigarette smoking in Latin America: a survey of eight cities. Bull Pan Am Health Organ 1975;9(4):328-344.

38. Lolio CAD, Souza JMPD, Santo AH, Buchalla CM. Prevalência de tabagismo em localidade urbana da região sudeste do Brasil. Rev Saude Publica 1993;27(4):262-265.

39. Serfaty EM, Kelmendi de Ustaran J, Andrade JH, Boffi-Boggero HJ, Masaútis AE, Foglia VL. Consumo de tabaco, alcohol, marihuana, cocaína y medicamentos no recetados. Acta Psiq Psicol Am Lat 2000;46(2):175-181.
40. Fassa AG. Trabalho infantil e saúde: perfil ocupacional e problemas músculo-esqueléticos [tese de doutorado]. Pelotas: Universidade Federal de Pelotas; 2000.

41. Malcon MC. Prevalência e fatores de risco para tabagismo em adolescentes: um estudo de base populacional, Pelotas, RS [dissertação de mestrado]. Pelotas: Universidade Federal de Pelotas; 2000.

42. Corrao MA, Guindon GE, Sharma N, Shokoohi DF, eds. The 11th World Conference on Tobacco or Health: tobacco control country profiles. Atlanta, Georgia: American Cancer Society; 2000.

43. Míguez HA, Pecci MC, Carrizosa A Epidemiología del abuso del alcohol y las drogas en el Paraguay. Acta Psiq Psicol Am Lat 1992; 38(1):19-29.

44. Horta BL, Calheiros P, Pinheiro RT, Tomasi E, Amaral KC. Tabagismo em adolescentes de área urbana na região Sul do Brasil. Rev Saude Publica 2001;35(2):159-164.

45. Muza GM, Costa MP. Aspectos sociofamiliares do consumo de tabaco por adolescentes escolares da rede privada do Distrito Federal. Revista ABP-APAL 1993;15(1):31-36.

46. Muza GM, Bettiol H, Muccillo G, Barbieri MA. Consumo de substâncias psicoativas por adolescentes escolares de Ribeirão Preto, SP (Brasil): II. Distribuição do consumo por classes sociais. Rev Saude Publica 1997;31(2):163-170.

47. Lauer RM, Akers RL, Massey J, Clarke WR. Evaluation of cigarette smoking among adolescents: the Muscatine study. Prev Med 1982; 11(4):417-428.

48. Woodward M, Tunstall-Pedoe H, Smith WCS, Tavendale R. Smoking characteristics inhalation biochemistry in the Scottish population. J Clin Epidemiol 1991;44(12):1405-1410.

Manuscrito recebido em 11 de fevereiro de 2002. Aceito em versão revisada em 26 de agosto de 2002

ABSTRACT Objective. To describe the prevalence of teenage smoking and the factors associated with smoking in this age group in South America.

\section{Prevalence of and risk factors for cigarette smoking among adolescents in South America: a systematic literature review}

Methods. Searches for articles concerning teenage smoking in Latin America were conducted in two bibliographic databases: MEDLINE (1966-2002) and Latin American and Caribbean Literature on the Health Sciences ("LILACS") (1982-2002). We also reviewed governmental and nongovernmental documents and Web sites. From the 315 articles that we identified, we considered 45 to be relevant for this paper. Results. In each of the countries that we studied, there was often a wide range found in prevalence levels, depending in part on the particular age range studied and on the definition of smokers that was used. The leading risk factor for teenage smoking was smoking among siblings and friends. Additional risk factors were poor academic performance, being older, male gender, having paid work, and having parents who were separated.

Conclusions. Additional population-based studies with teenagers should be carried out, and more uniform criteria for defining smokers should be developed.

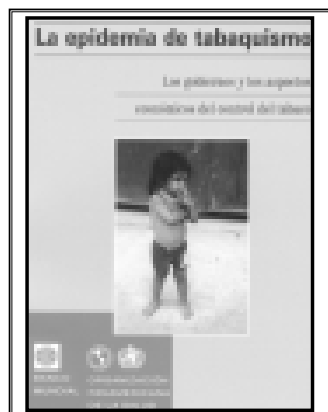

$2000 \cdot 133 \mathrm{pp}$.

ISBN 9275315779

Código: PC 577

Precio: US\$ 25.00/

US\$ 18.00 en América Latina y el Caribe

\section{La epidemia de tabaquismo: Los gobiernos y los aspectos económicos del control del tabaco}

Este informe ofrece una evaluación de los aspectos económicos del control del tabaco en el que se revisa la experiencia internacional. Las conclusiones del mismo son que la elevación de los impuestos sobre el tabaco puede salvar millones de vidas y aumentar, al mismo tiempo, los ingresos estatales a mediano plazo, y que otras medidas no relacionadas con el precio, como la prohibición completa de la publicidad y de la promoción de los cigarrillos, también reducirían de manera importante el consumo de tabaco. Se examinan los efectos que las políticas de control del tabaco tienen sobre el empleo y se llega a la conclusión de que la mayoría de los países no sufrirían pérdidas permanentes de puestos de trabajo.

El informe examina también los costos de las políticas de control y establece un calendario para la acción de los gobiernos, incluida la ayuda a los cultivadores de tabaco más pobres. Señala asimismo el papel que deben desempeñar los organismos internacionales en la reducción de la carga evitable de muertes prematuras y discapacidades relacionadas con el tabaco.

Para adquirir esta publicación: http://publications.paho.org; E-mail: paho@pmds.com; Fax: (301) 209-9789; Tel: (301) 617-7806; (1-800) 472-3046 